\title{
A Comparative Analysis And Applications Of MULTI WAVELET TRANSFORM IN IMAGE DENOISING
}

\author{
Smriti Bhatnagar ${ }^{1}$ and R.C.Jain ${ }^{2}$ \\ ${ }^{1}$ Department of Electronics and Communication Engineering, Jaypee Institute Of \\ Information Technology, NOIDA, India
}

\begin{abstract}
In the era of telemedicine a large amount of medical information is exchanged via electronic media mostly in the form of medical images, to improve the accuracy and speed of diagnosis process. Medical Image denoising has the basic importance in image analysis as these algorithm and procedures affects the efficacy of medical diagnostic. In this paper focus is on Multi wavelets based Image denoising techniques, because they provide the possibility of designing wavelets systems which are orthogonal, symmetric and compactly supported, simultaneously. Performance of Discrete Multi Wavelet Transform and Discrete Wavelet Transform based denoising methods are compared on the basis of PSNR.
\end{abstract}

\section{KEYWORDS}

Multiwaveletst; Wavelets; denoising.

\section{INTRODUCTION}

Recently, a lot of interest and research work is appearing in the development of denoising algorithms for medical images in wavelet domain .Multi wavelets are the new paradigm added in this domain. Multi wavelets present the advantages of simultaneous orthogonality, symmetry and short support but at the cost of pre filtering and post filtering. Pre filtering is the process of vectorizing the input before application of multi wavelets. Several methods are there to tackle this problem $[2,3,4,5,10]$. The postfilter, on the other hand, maps the data from multiple channels to one channel again.

In Multi wavelets case, more than one scaling and wavelet functions are used to represent a signal. Multi wavelet decomposition can be implemented with filter banks as the case with scalar wavelets but filter coefficients in this case are matrices instead of scalar values.

Instead of thresholding individual multi wavelet coefficients similar with scalar wavelets, coefficient vectors are considered and thresholding operation is applied to the whole vector.

In this paper the multi wavelets based denoising technique is discussed and its performance is compared on the basis of Peak Signal to Noise Ratio (PSNR) and Mean Square Error (MSE) with scalar wavelet based denoising technique.

Section II gives mathematical preliminaries and notations associated with multi wavelet bases. Discussion on chosen multi wavelets GHM and CL is also given in section II.

DOI: $10.5121 /$ ijci.2015.4215 
International Journal on Cybernetics \& Informatics (IJCI) Vol. 4, No. 2, April 2015

Section III comprises of pre filtering and post filtering techniques for implementation of multiwavelet transform. Section IV characterizes the Multi wavelet Filter Banks. With the help of filter banks discussed in section IV, in section V, denoising schemes with multi wavelets are discussed and comparison of this scheme is done with other scalar wavelet techniques.

\section{Mathematical Preliminaries}

Important mathematical concepts related with multi wavelets are discussed here.

\subsection{Multiwavelets}

Characterization of multi wavelets are done with multiple scaling functions and multiple wavelet functions[1,2].

Denoting the scaling functions in the vector form as $\quad \phi(t)=\left[\phi_{0}(t), \phi_{1}(t), \ldots \ldots \ldots \phi_{r-1}(t)\right]^{T}, \phi(t)$ is multiscaling function and $\mathrm{T}$ is transpose.$\phi_{i}(t)$ is $\mathrm{i}^{\text {th }}$ scaling function. Similarly denoting the wavelet functions in the vector form as $\psi(t)=\left[\psi_{1}(t), \psi_{2}(t), \ldots \ldots \ldots \psi_{r}(t)\right]^{T}, \psi(t)$ is multiwavelet function and $\mathrm{T}$ is transpose. $\psi_{i}(t)$ is $\mathrm{i}^{\text {th }}$ wavelet function. The scaling space

$$
V_{j}=\underset{k}{\operatorname{span}}\left\{\phi_{0}\left(2^{j} t-k\right), \ldots, \phi_{r-1}\left(2^{j} t-k\right)\right\}
$$

and the wavelet space

$$
W_{j}=\underset{k}{\operatorname{span}}\left\{\psi_{0}\left(2^{j} t-k\right), \ldots, \psi_{r-1}\left(2^{j} t-k\right)\right\}
$$

Dilation equation in traditional scalar wavelet is obtained from the nesting condition $V_{j} \subset V_{j+1}$.Same is true for multi wavelets.The matrix dilation or refinement equation for multi wavelets can be represented as

$$
\phi(t)=\sqrt{2} \sum_{k} H_{k} \phi[2 t-k]
$$

and the wavelet equation as

$$
\psi(t)=\sum_{k} G_{k} \psi[2 t-k]
$$

Here the pair of filters $H_{k}$ and $G_{k}$ are low pass multi wavelet filter banks and high pass multi wavelet filter banks respectively. These are rxr matrix filters instead of scalar filters in case of wavelets. In general $r=2$ is considered but it could be any value greater than 1 . For $r=2$, these filters will be $2 \times 2$ matrix filters operating on two input data stream. Filter coefficients are matrices in the case of multi wavelets and provide more degree of freedom as compared to scalar wavelets. For arbitrary chosen value of $r$, multi wavelets are known as of multiplicity $r$. 


\subsection{GHM and CL Multiwavelets}

Alpert constructed the polynomial multi wavelets, and used them as basis for some operators. After that Geronimo, Hardin and Massopust had done the construction of multi scaling function for the first time with the help of fractal interpolation[2].

For multiplicity of 2 , eq (5) represents the relation of scaling functions $\varphi_{1}(t)$ and $\varphi_{2}(t)$ with low pass filter coefficients and similarly eq (6) represents the relation of wavelet functions $\psi_{1}(\mathrm{t})$ and $\psi_{2}(\mathrm{t})$ with high pass filter coefficients.

$$
\begin{aligned}
& \left\{\Phi(t)=\left[\begin{array}{l}
\varphi_{1}(t) \\
\varphi_{2}(t)
\end{array}\right]=\begin{array}{l}
H_{0} \Phi(2 t)+H_{1} \Phi(2 t-1) \\
\\
+H_{2} \Phi(2 t-2)+H_{3} \Phi(2 t-3)
\end{array}\right\} \\
& \left\{\Psi(t)=\left[\begin{array}{l}
\psi_{1}(t) \\
\psi_{2}(t)
\end{array}\right]=\begin{array}{l}
G_{0} \Phi(2 t)+G_{1} \Phi(2 t-1) \\
+G_{2} \Phi(2 t-2)+G_{3} \Phi(2 t-3)
\end{array}\right\}
\end{aligned}
$$

Low pass filter coefficients $H_{k}$ 's and high pass filter coefficients $G_{k}$ 's for GHM are listed below as eq (8) to eq (9)

$$
\begin{array}{cc}
H_{0} & =\left[\begin{array}{cc}
3 / 5 & 4 \sqrt{2} / 5 \\
-1 / 10 \sqrt{2} & -3 / 10
\end{array}\right], \quad H_{1}=\left[\begin{array}{cc}
3 / 10 & 0 \\
9 \sqrt{2} / 40 & 1 / 2
\end{array}\right] \\
H_{2} & =\left[\begin{array}{cc}
0 & 0 \\
9 \sqrt{2} / 40 & -3 / 20
\end{array}\right], \quad H_{3}=\left[\begin{array}{cc}
0 & 0 \\
-\sqrt{2} / 40 & 0
\end{array}\right] \\
G_{0} & =\left[\begin{array}{cc}
3 / 10 & 2 \sqrt{2} / 5 \\
-\sqrt{2} / 40 & -3 / 20
\end{array}\right] \quad G_{1}=\left[\begin{array}{cc}
3 / 10 & 2 \sqrt{2} / 5 \\
-\sqrt{2} / 40 & -3 / 20
\end{array}\right] \\
G_{2} & =\left[\begin{array}{cc}
3 / 10 & 2 \sqrt{2} / 5 \\
-\sqrt{2} / 40 & -3 / 20
\end{array}\right] \quad G_{3}=\left[\begin{array}{cc}
3 / 10 & 2 \sqrt{2} / 5 \\
-\sqrt{2} / 40 & -3 / 20
\end{array}\right]
\end{array}
$$

GHM multi wavelet possess wonderful properties like its scaling functions are symmetric with short support $[0,1]$ and $[0,2]$. Scaling functions are orthogonal and multi wavelet functions are symmetric/anti symmetric pair. This multi wavelet provides second order approximation[6].

Another interesting multi wavelet CL of multiplicity $\mathrm{r}$ posses [7] the low pass filter coefficients $\mathrm{H}$ k's and high pass filter coefficients $G_{k}$ 's listed below as eq(9) to eq(10). 
International Journal on Cybernetics \& Informatics (IJCI) Vol. 4, No. 2, April 2015

$$
\begin{array}{ll}
H_{0} & =\left[\begin{array}{cc}
1 / 4 & 1 / 4 \\
-\sqrt{7} / 8 & -\sqrt{7} / 8
\end{array}\right] \quad H_{1}=\left[\begin{array}{lr}
1 / 2 & 0 \\
0 & 1 / 4
\end{array}\right] \quad H_{2}=\left[\begin{array}{cc}
1 / 4 & -1 / 4 \\
-\sqrt{7} / 8 & -\sqrt{7} / 8
\end{array}\right] \\
G_{0} & =\left[\begin{array}{cc}
-1 / 4 & -1 / 4 \\
1 / 8 & 1 / 8
\end{array}\right] \quad G_{1}=\left[\begin{array}{lr}
1 / 2 & 0 \\
0 & \sqrt{7} / 20
\end{array}\right] \quad G_{2}=\left[\begin{array}{cc}
-1 / 4 & 1 / 4 \\
-1 / 8 & 1 / 8
\end{array}\right]
\end{array}
$$

The CL multi wavelets also has some important properties like both of its scaling functions have short support of $[0,2]$. Scaling functions and wavelet functions are symmetric and anti symmetric orthonormal pairs.

Such important properties cannot be fulfilled simultaneously in case of scalar wavelets. Filter coefficients in multi wavelet cases are matrices and provide more degree of freedom in designing and applications so the better performance is expected than scalar wavelets [8].

Graphical representation of scaling and wavelet functions for GHM is given in Figure 1.
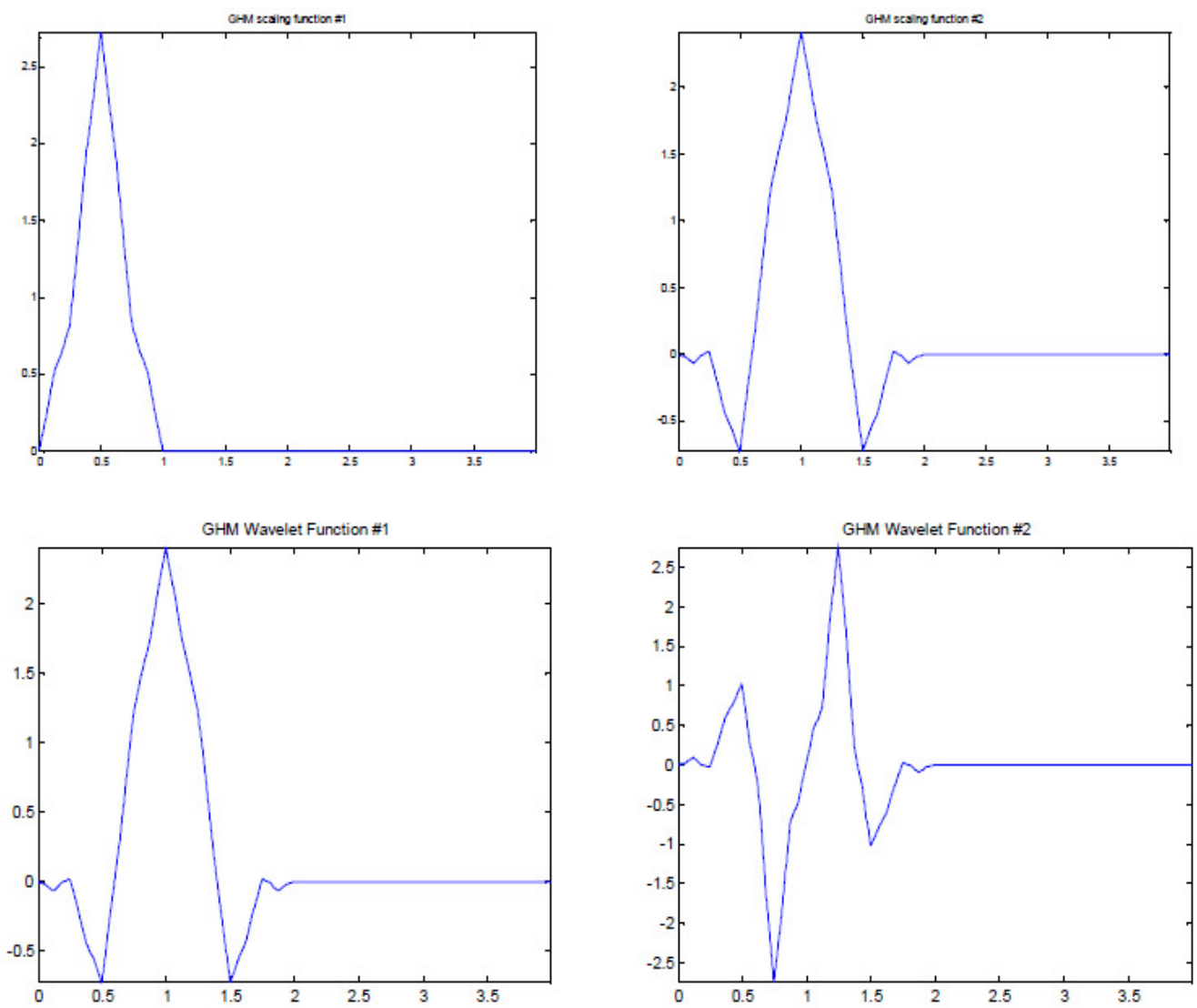

Figure. 1 Scaling functions $\varphi_{1}(\mathrm{t})$ and $\varphi_{2}(\mathrm{t})$ and wavelet functions $\psi_{1}(\mathrm{t})$ and $\psi_{2}(\mathrm{t})$ for GHM Multi wavelet Graphical representation of scaling and wavelet functions for CL is given in Figure 2. 


\section{Prefiltering And Postfiltering}

Before applying multi wavelets, input data has to be pre processed or vectorized, which is known as pre filtering or initialization of multi wavelets in literature. With the help of pre filters multiple data streams are generated. Generally two types of pre filtering process are used, named as repeated row pre filtering and critically sampled scheme[10]. Repeated row pre filtering is not suited for data compression as it introduces redundancy. In critically sampled scheme, multiple rows of data are generated by sampling the input data and distributing them to different rows [9]. Post filtering operation is mapping again from multiple streams to a single data stream or reconstruction. Basically perfect reconstruction properties has to be satisfied by set of preprocessing and post processing filters. Performance and design of multi wavelets depends a lot on selection of these filters [12].
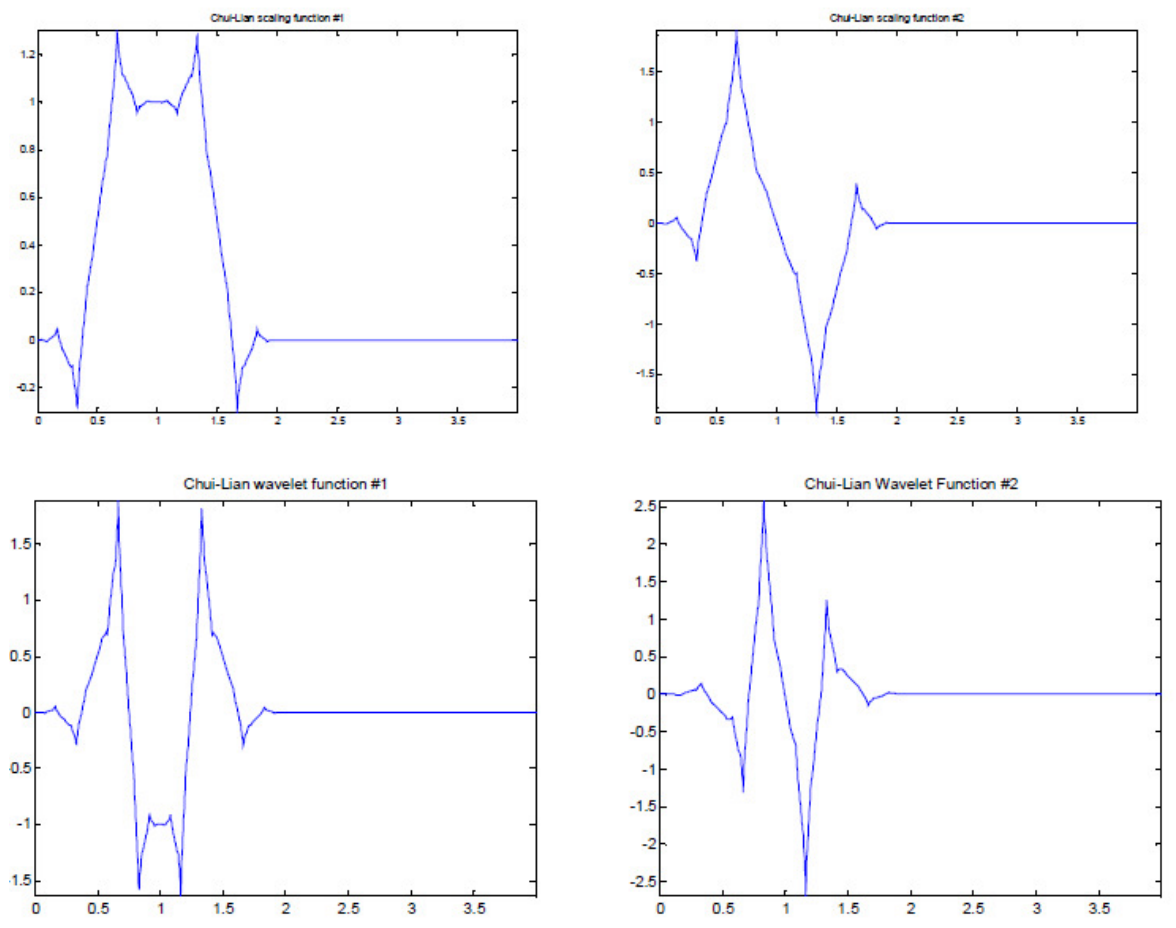

Figure.2.Scaling functions $\varphi_{1}(\mathrm{t})$ and $\varphi_{2}(\mathrm{t})$,Wavelet functions $\quad \psi_{1}(\mathrm{t})$ and $\psi_{2}(\mathrm{t})$ for CL Multi wavelet

\section{Multiwavelet Filter Banks}

Multi wavelets of multiplicity ' $r$ ' require ' $r$ ' input streams to the multi wavelet filter banks. A multi wavelet filter banks has taps that are rxr matrices. Coefficients for the low pass filter bank $H_{k}$ are given by four rxr matrices and the same is true for High pass filters $G_{k}$. Here coefficients of high pass filter $\mathrm{G}_{\mathrm{k}}$ cannot be obtained by flipping low pass filter coefficients as in it is done in scalar wavelets [14]. It has to be designed separately. Finally $r$ channel rxr matrix filter bank operates on $r$ input data streams and generate $2 r$ output streams which are then down sampled by 2. Every row of multifilters are a combination of $r$ ordinary filters each operating on different data stream. Figure 3 represents a multi wavelet filter bank[10]. 
Similar to the scalar wavelet, the multi wavelet theory is also based on multi resolution analysis. If we decompose an image using a scalar wavelet to single level of decomposition, resultant data will correspond to four sub band of low pass /high pass filter in both the dimensions. Data in LH sub band is output from high pass filtering of rows first and then low pass filtering of column. For multi wavelets with multiplicity $r=2$, will have two sets of scaling coefficients. In case of multi wavelets subscript 1 and 2 along with $\mathrm{L}$ and $\mathrm{H}$ corresponds to the channel 1 and 2 respectively. For example $\mathrm{L}_{1} \mathrm{H}_{2}$ represent the data from the second channel high pass filter in the horizontal direction and first channel low pass filter in the vertical direction. Thus after first level of decomposition of an image with multi wavelets will correspond to 16 sub band shown in Fig 4 and multi wavelet decomposition up to third level is shown in Figure 5.

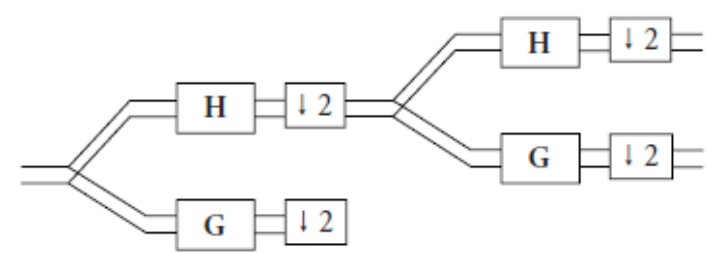

Figure 3. Multi wavelet filter bank

\begin{tabular}{|c|c|}
\hline L L & L H \\
\hline H L & H H \\
\hline
\end{tabular}

(a)

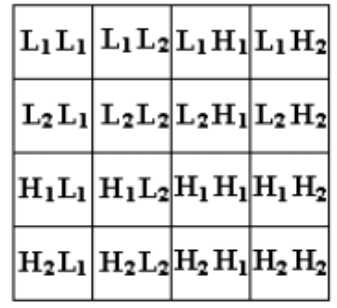

(b)

Figure 4. First level of decomposition (a) using scalar wavelets (b) using multiwavelet

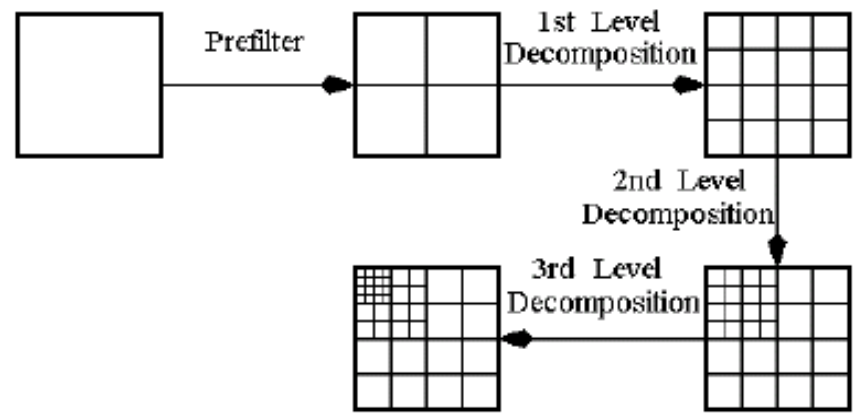

Figure 5.Multiwavelet decomposition up to third level

\section{DeNOISING SCHEME}

Steps for de noising scheme for multi wavelets are first to use a pre filter to convert the original data in to multiple streams. Then take the multi wavelet transform of this multiple stream. Apply the thresholding scheme on coefficients and then perform inverse multi wavelet transform of the thresholded data. In the last apply a post filter to get the de noised data. 
International Journal on Cybernetics \& Informatics (IJCI) Vol. 4, No. 2, April 2015

Signal denoising techniques are based on the idea of thresholding the wavelet coefficients. Wavelet coefficients having small absolute value are considered to have mostly noise and very fine details of the signal. In contrast, the important information is represented by the coefficients having large absolute value. Removing the small absolute value coefficients and then reconstructing the signal should produce signal with lesser amount of noise. In case of multi wavelets, thresholding whether hard or soft is applied on whole vector [11]. The thresholding of the subband coefficients

are done by two ways(a) Hard theresholding function is defined as

$$
F(x)=x . I(|x|>T)
$$

And (b) Soft threshold shrinks coefficients above the threshold in absolute value.

$$
F(x)=\operatorname{sgn}(x)(|x|-t) I(|x|>T)
$$

The only difference between the hard and the soft thresholding procedure is in the choice of the nonlinear transform on the empirical wavelet coefficients [13]. Threshold $\mathrm{T}$ to be set to the known noise level.

\section{Parameters For Comparison}

\subsection{Mean Square Error (MSE)}

MSE, which for two $\mathrm{m} x \mathrm{n}$ monochrome images $\mathrm{x}$ and $\mathrm{y}$ where one of the images is considered noisy approximation of the other and is defined as in equation (13)

$$
M S E=\frac{1}{m n} \sum_{k=0}^{m-1} \sum_{l=0}^{n-1}[X(k, l)-Y(k, l)]^{2}
$$

\subsection{Peak Signal to Noise Ratio (PSNR)}

PSNR is the peak signal to noise ratio in decibels $(\mathrm{dB})$. The PSNR is only meaningful for data encoded in terms of bits per sample bits per pixel. For example an image with 8 bits per pixel contains integers from 0-255. PSNR is given by the equation (14)

$$
\text { PSNR }=20 \log _{10}\left(2^{\mathrm{B}}-1\right) / \mathrm{MSE}
$$

Comparison is based on this parameter values for de noising schemes using different wavelet/multi wavelets. Here B = No. of bits

\section{EXPERIMENTAL RESULTS}

In experiment, test images used are de noised using GHM, CL and DB4. DB4 scalar wavelet is used to compare the results of denoising of medical images with multi wavelets like GHM and CL . Reason behind the selection of DB4 is its properties of orthogonality , 4 coefficients in the dilation equation with 2 vanishing moments which are very similar to GHM multiwavelet. Computatinal results are given in Table 1. 
International Journal on Cybernetics \& Informatics (IJCI) Vol. 4, No. 2, April 2015

Table 1 : Computational Results

\begin{tabular}{|c|c|c|}
\hline $\begin{array}{c}\text { Type of } \\
\text { Wavelets/Multiwavelets }\end{array}$ & $\begin{array}{c}\text { Type of } \\
\text { thresholding }\end{array}$ & PSNR \\
\hline DB4 & Soft & 16.4571 \\
\hline GHM & Soft & 19.9876 \\
\hline CL & Soft & 17.5672 \\
\hline DB4 & Hard & 13.4042 \\
\hline GHM & Hard & 15.7634 \\
\hline CL & Hard & 14.4782 \\
\hline
\end{tabular}
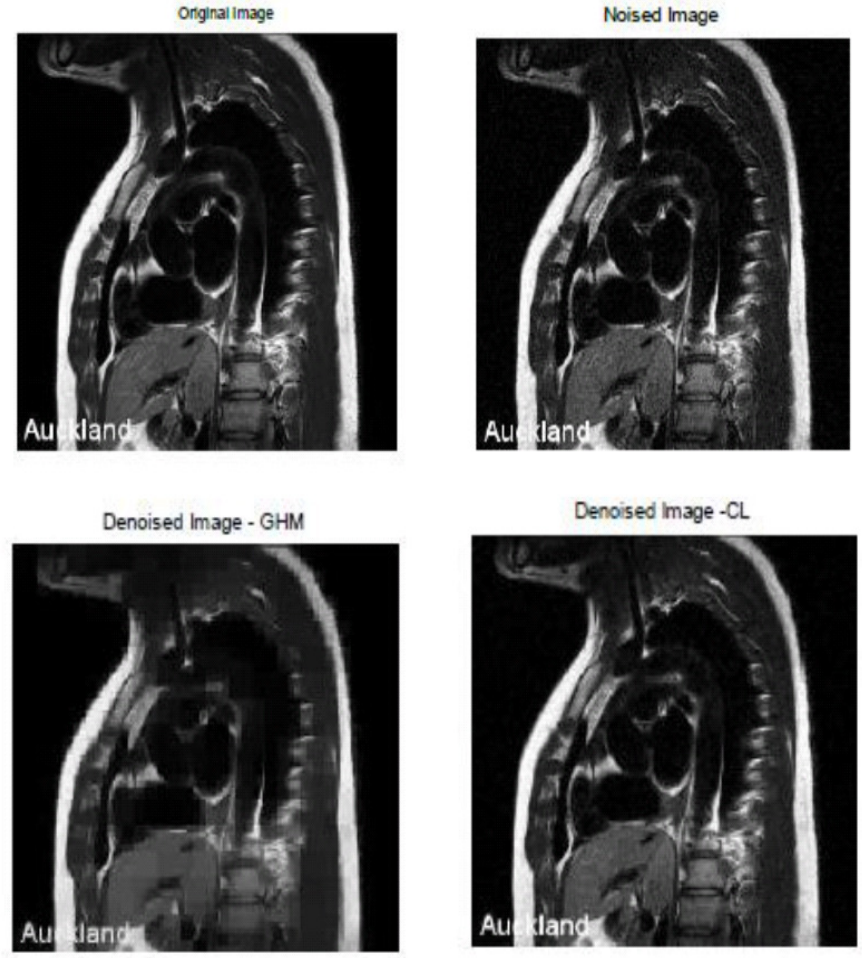

Figure 6.Denoising of Images using different Multi wavelets Transform

\section{CONCLUSION}

Results shown there in Table 1. and Figure.6, give us a fair idea that multi wavelets like GHM and CL gives better results than scalar wavelets in application of denoising of medical images. It opens the door of lots of future work in area of medical image denoising with different thresholding techniques associated with these multiwavelets. 


\section{ACKNOWLEDGEMENTS}

Medical images used here are taken form freely available medical images provided by Auckland MRI Research Group.

\section{REFERENCES}

[1] T. N. T. Goodman and S. L. Lee, "Wavelets of multiplicity r," Trans. Amer. Math. Soc., vol. 342, pp. 307-324, 1994.

[2] M. Cotronei, L. B. Montefusco, and L. Puccio, "Multiwavelet analysis and signal processing," IEEE Trans. Circuits Syst. II, vol. 45, no. 8, pp. 970-985, 1998.

[3] V. Strela, Multiwavelets: theory and applications, Ph.D. thesis, Massachusetts Institute of Technology, Boston, Mass, USA, 1996.

[4] D. P. Hardin and D. Roach, "Multiwavelet prefilters I: Orthogonal prefilters preserving approximation order $p \leq 2$," IEEE Trans. Circuits Syst. II, vol. 45, no. 8, pp. 1106-1112,1998.

[5] V. Strela, P. Heller, G. Strang, P. Topiwala, and C. Heil, "The application of multiwavelet filter banks to signal and image processing," IEEE Trans. Image Processing, vol. 8, no. 4, pp. 548$563,1999$.

[6] J. Geronimo, D. Hardin, and P. R. Massopust, "Fractal functions and wavelet expansions based on several scaling functions," J. Approx. Theory, vol. 78, no. 3, pp. 373-401, 1994.

[7] C. K. Chui and J. A. Lian, "A study of orthonormal multi-wavelets," Cat report 351, Texas A\&M University, Texarkana,Tex, USA, 1995.

[8] J. Qumar and S. Gupta, "Performance Measure of Despeckling of SAR Image using Multiwavelets" NCC 2009, pp.357- 361, 2009.

[9] G. Strang and V. Strela, "Short wavelets and matrix dilation equations," IEEE Trans. Signal Processing, vol. 43, no. 1, pp.108-115, 1995.

[10] X. G. Xia, J. S. Geronimo, D. P. Hardin, and B. W. Suter, "Design of prefilters for discrete multiwavelet transforms," IEEETrans. Signal Processing, vol. 44, no. 1, pp. 25-35, 1996.

[11] T. R. Downie and B. W. Silverman, "The discrete multiple wavelet transform and thresholding methods,” IEEE Trans. Signal Processing, vol.46, pp. 2558-2561, Sept. 1998.

[12] T. Nguyen and G. Strang, Wavelets and Filter Banks. Cambridge,MA: Wellesley-Cambridge, 1995.

[13] S.G.Chang, B.Yu, and M.Vitterli , "Adaptive Wavelet thresholding for image denoising and compression" IEEE Transactions on Image Processing, vol. 9, no. 9, pp 1532-1546,Sept. 2000.

[14] M.B.Martin, and A.E.Bell "New Image Compression Techniques using multiwavelets and multiwavelet packets" IEEE Transactions on Image Processing, vol. 10, no. 4, pp 500-510,April 2001.

\section{AUTHORS}

Smriti Bhatnagar is faculty at ECE Department, Jaypee Institute of Infor mation Technology, NOIDA. She has done M.E., from MNIT, Allahabad. Her research interests are System Design \& Digital Signal Processing.

Dr.R.C.Jain is presently Professor \& Head, ECE Department, Jaypee Institute of Information Technology, NOIDA. He has done M. Eng., and Ph. D., Electrical Engineering, from University of Alberta, Canada. His research area is Wireless Communication Systems.

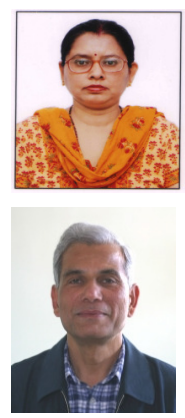

